\title{
Evaluation of Inferior Pedicle Therapeutic Mammoplasty as a Primary Procedure for Upper Quadrants Early Breast Cancer $^{*}$
}

\author{
Adel Denewer ${ }^{\#}$, Waleed Elnahas, Osama Hussein, Ashraf Khater, Wael El-Sadda, \\ Khaled M. Abouelkher \\ Mansoura Oncology Center, Mansoura, Egypt \\ Email: \#adeldenewer@yahoo.com
}

Received March 31, 2013; revised May 1, 2013; accepted May 10, 2013

Copyright (C) 2013 Adel Denewer et al. This is an open access article distributed under the Creative Commons Attribution License, which permits unrestricted use, distribution, and reproduction in any medium, provided the original work is properly cited.

\begin{abstract}
Background: The treatment of breast cancer in large breast patients represents a great challenge to both surgical oncologist and radiation oncologist. The aim of this study is to evaluate the outcome of inferior pedicle therapeutic mammoplasty in large-breasted patients with upper quadrants early breast cancer. Methods: Thirty five large-breasted patients with early breast cancer were included in this study. Simultaneous bilateral inferior pedicle therapeutic mammoplasty was performed. Results: The age of the patients is ranged from 36 to 61 (median 46) years and tumour size is ranged from one to three and half $\mathrm{cm}$. The weight of tissue removed is ranged from $350 \mathrm{gm}$ to $780 \mathrm{gm}$ and the tumour safety margins are ranged from three to eight $\mathrm{cm}$. Wound dehiscence was the commonest post operative complications and six patients were affected (17.6\%). The cosmetic outcome was excellent in 22 patients (64.5\%), nine patients (26.5\%) showed good results, two patients (6\%) were satisfactory and one patient (3\%) showed poor result. The follow up period is ranged from 6 to 42 months with one case (3\%) of systemic metastasis. Conclusion: Inferior pedicle therapeutic reduction mammoplasty for upper quadrants early breast cancer in large breasted women is a surgically and oncologically safe procedure, and it carries a satisfactory aesthetic outcome.
\end{abstract}

Keywords: Breast Cancer; Conservative Surgery; Reduction Therapeutic Mammoplasty

\section{Introduction}

The surgical management of breast cancer has been based on two main options; either tumour resection with safety margin (breast-conserving surgery) (BCS) or the standard mastectomy with or without reconstruction [1] Breast conservation surgery (BCS) is oncologically safe as demonstrated by robust level one evidence from well conducted randomized controlled trials which show equivalence with mastectomy in terms of survival [2]. After traditional breast conservation therapy, $20 \%-30 \%$ of patients were reported to have poor cosmetic results [3].

Patients with macromastia treated with breast conservation therapy develop more complications and unacceptable cosmesis due to heterogeneous distribution of radiotherapy dose and suboptimal positioning of the breast between treatment sessions [4,5].

\footnotetext{
"The authors have no financial and personal relationships with other people or organizations that could inappropriately influence this work. "Corresponding author.
}

Volume displacement with well-established breast reduction techniques, or replacement, maximizes the volume of tissue that can be excised, resulting in effective local control whilst maximizing aesthetic outcomes [6].

Therapeutic mammoplasty is indicated in women where the tumour lies within the excision pattern of an established breast reduction technique, and for cases in which contralateral breast reduction to achieve symmetry is viewed as a positive outcome [7].

The aim of this paper is to report our results for inferior pedicle therapeutic mammoplasty reduction technique in patients with early breast cancer and have large breast.

\section{Patients and Methods}

Between august 2009 to October 2012, 35 large-breasted women with upper quadrants early stage breast cancer at outpatient's clinic mansoura oncology center were included in this study. All patients fulfilled the standard 
criteria for breast conservation therapy. Oncologic exclusion criteria were multicentric carcinoma, inflammatory breast cancer, inability to obtain tumour-free safety margins after reasonable attempts, contraindication to radiotherapy. Non-oncologic exclusion criteria were small breast size, centrally located tumours, comorbidity, and the patient own preference. The steps of the procedure were discussed with all patients and informed consent was obtained. Table 1 summarizes the patients and tumour characteristics.

Preoperative markings while the patient at upright position. The inferior pedicle was used for all patients (Figure 1).

Intraoperatively the tumour was resected first using the incisions designed preoperatively to enable the optimum oncological excision and the specimen was examined by frozen section in order to assess the safety margins.

The tumour bed was marked by clips.

Table 1. Patients and tumour characteristics.

\begin{tabular}{lc}
\hline Patients age (Year) & $38-61$ \\
Range & 46 \\
Median & \\
Tumour pathology & 29 \\
Invasive duct carcinoma & 4 \\
Medullary carcinoma & 2 \\
Mucinous carcinoma & \\
Tumour stage & 8 \\
pT1 & 27 \\
pT2 & 11 \\
pN0 & 24 \\
pN1 & \\
Grading & 6 \\
G1 & 25 \\
G2 & 4 \\
G3 & \\
Tumour location & 30 \\
Upper outer quadrant & 5 \\
Upper inner quadrant & \\
\hline
\end{tabular}

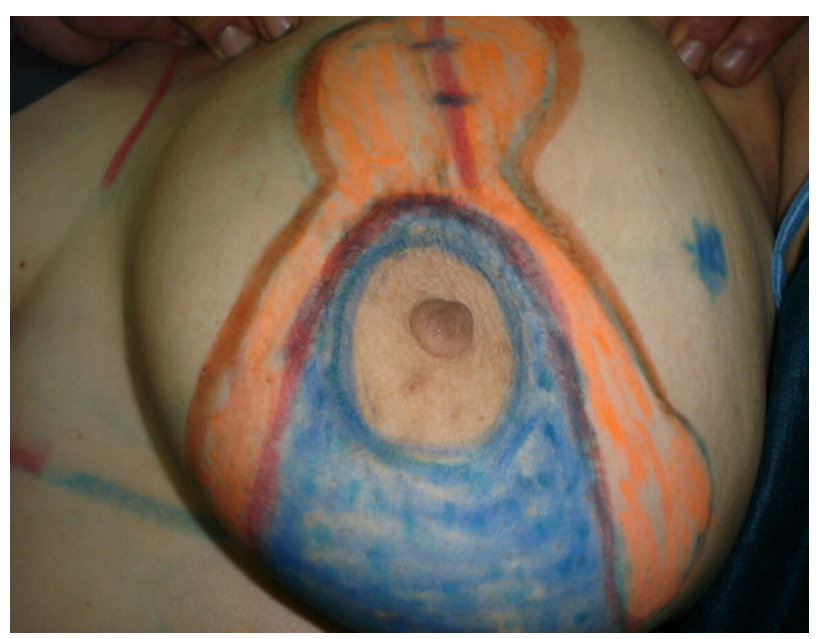

Figure 1. Preoperative marking of inferior pedicle.
De-epithelialization of the skin in the lower part of the breast was performed keeping the width of the inferior pedicle more than $8 \mathrm{~cm}$. resection of tissues on either side of the pedicle. The flaps are then approximated along the inframammary fold and up along the vertical incision with interrupted and running 3 - 0 absorbable monofilament sutures. A circular defect is created in the midline at the apex of the breast. The areola is then sutured into position to complete the procedure (Figure 2).

The axilla was cleared through a separate transverse incision in the axillary hair line removing levels 1 and 2 .

In our series, we started first with the diseased breast, and after assessment of adequate free tumour safety margins, the same procedure in the contralateral breast was performed to obtain symmetry (Figures 3 and $\mathbf{4}$ ).

All patients were referred to the clinical oncology and nuclear medicine department where they received the radiotherapy and adjuvant chemotherapy according to the stage of the tumour. Assessment: Table 2.

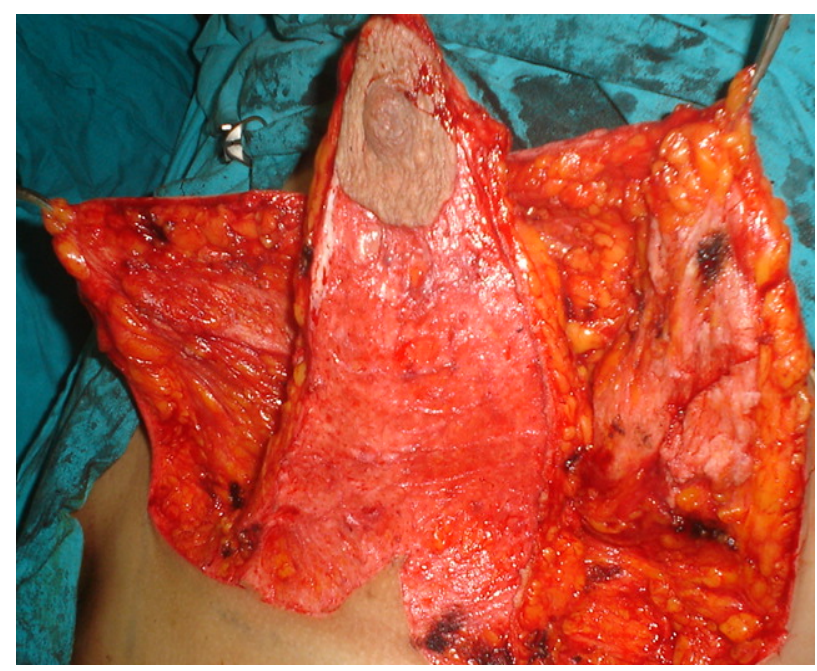

Figure 2. De-epithelialization of the dermo-glandular flap.

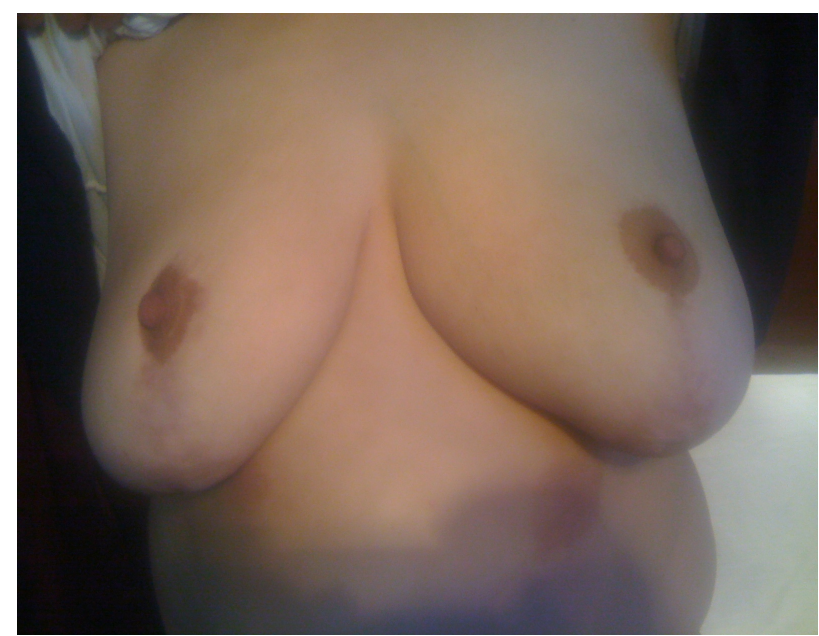

Figure 3. Post-operative view after one and half months. 


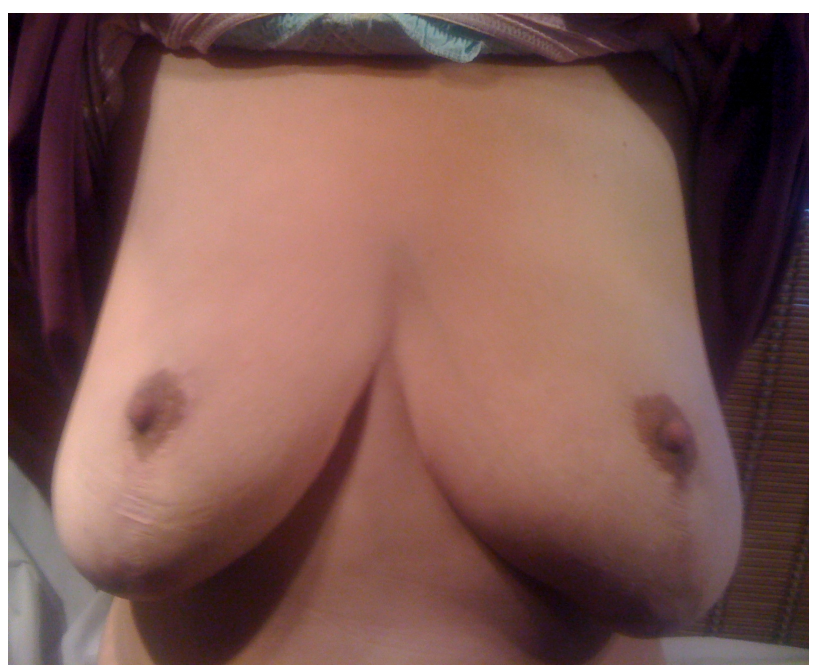

Figure 4. Post-operative view after one year.

Table 2. Overall complication rate.

\begin{tabular}{ll}
\hline Complications & Number \\
\hline Minor skin necrosis & $4(11.7 \%)$ \\
Wound dehiscence & $6(17.6 \%)$ \\
Infection & $1(3 \%)$ \\
Fat necrosis & $1(3 \%)$ \\
Partial areala necrosis & $1(3 \%)$ \\
Total areala necrosis & $0(0 \%)$ \\
Seroma & $5(14.7 \%)$ \\
\hline
\end{tabular}

\subsection{Procedure Related Complications}

Early post operative complications: During the hospital stay (maximum of 6 days), patients were assessed for the onset of wound infection, dehiscence, nipple and areola necrosis, and haematoma formation.

Late postoperative complications: For one month postoperatively in the outpatient visits, assessment was made for wound infections, persistent seroma in the breast and axilla.

\subsection{Aesthetic Outcome}

The cosmetic evaluation was performed 6 month postoperatively by simple score both subjectively and objectively and the mean was recorded. The score assessment was performed using a grading system. A score of 5 to 1 ( 5 = excellent; 4 = good; 3 = satisfactory; 2 = poor; $1=$ very poor) was given after evaluation of the following parameters: symmetry of the breasts, shape of the breast, symmetry of NAC placement, ipsilateral and contralateral scars [8].

\subsection{Oncologic Outcome}

All patients were evaluated in the outpatient clinics for local recurrence. Follow up was every two weeks for one month, monthly for 6 month, every 3 month for one year, every 6 month for 2 years and then yearly. Bilateral breast ultrasound was performed every 3 - 6 months. Mammograms were done for all the patients annually. MRI was done when mammography revealed suspicious data.

\section{Results}

The age of the patients ranged from 38 to 61 (median 46).all patients had tumours at the upper quadrant. The size of the tumour ranged from 1 to $3.5 \mathrm{~cm}$. most of the patients were diagnosed as having infiltrating ductal carcinoma (29 patients, 82.8\%). The weight of tissues removed ranged from $350 \mathrm{~g}$ to $780 \mathrm{~g}$. The tumour safety margins ranged from 3 to $8 \mathrm{~cm}$.

Only one patient had infiltrated margin at frozen section after 2 attempts of excision and mastectomy was performed (conversion rat about 3\%).

Wound dehiscence was the commonest post operative complications and affect 6 patients (17.6\%). one patients was due to partial areala necrosis and managed by debridement and secondary sutures while the other 5 had dehiscence at the lower part of the longitudinal scar at its confluence with the inframammary fold 4 patients were minor and managed conservatively. The fifth patient has secondary infection and was managed by secondary suturing after infection control.

Seroma developed in 5 patients (14.7\%) and was treated by frequent aspiration.

One patient developed a small firm area at the cranial end of the areola along the suture line 10 month postoperatively and was investigated by mammography and trucut biopsy revealing traumatic fat necrosis that was surgically excised.

Six month postoperatively, the cosmetic was evaluated. 22 patients (64.5\%) were excellent results, 9 women (26.5\%) showed good results, two patient (6\%) were satisfactory and one patient (3\%) showed poor result.

One patient after 18 month post operatively developed bone metastasis without any local recurrence and referred to medical oncology and nuclear medicine for completion of treatment.

No local recurrence or systemic metastasis was noticed in the other 33 patients during the follow up period which ranged from 6 to 42 months

\section{Discussion}

The conventional options of mastectomy or breast conservations therapy in patients with large pendulous breasts have many drawbacks ranging from difficulties for the radiation oncologist when breast conservation was chosen to unacceptable and uncomfortable asymmetry when unilateral mastectomy was performed.

Although breast conservation can be easily achieved 
with negative margins in this population of women with macromastia there are associated problems of chronic radiation induced pain, fibrosis and poor cosmetic result after radiotherapy [9].

Oncoplastic surgery has proven to be an oncologically safe procedure, with rates of local recurrence, metastasis and death comparable to breast conservation surgery [10].

For patients with significant macromastia, reduction mammoplasty may offer the best treatment outcome and superior quality of life. It should be strongly considered in this subgroup of patients because the new, smaller breast receives a more homogeneous radiation treatment. [11].

In this study, inferior pedicle reduction therapeutic mammoplasty was used for management of early breast cancer in large breasted women, and we consider it to be a more conservative and less radical procedure.

Gulcelik et al. performed therapeutic mammoplasty to 101 patients with breast cancer associated with macromastia and used inferior pedicle for most of his patient and reported that there were 5 seromas (5\%), 2 hematomas (2\%), 3 surgical site infection (3\%), 3 minor incisional dehiscence (3\%), 4 delayed wound healing (4\%), 1 areola necrosis (1\%), and 1 major wound dehiscence (1\%) [12].

In the present study the rate of seroma was high (14.7\%) this may be due to our routine use of diathermy in the dissection. The wound dehiscence occurred in 6 patients (17.6\%) and this was the most common complication. Delayed inverted-T-incision wound healing was the most common and happened in the early cases and avoided in later cases by leaving a small triangle of skin in the midline above the inframammary fold. Fitzel et al. reported that skin necrosis and wound dehiscence are the most often reported complications after therapeutic mammoplasty [13]. Partial areola necrosis encountered in one patient (3\%) while wound infection occurred in another one patient (3\%).

Munhoz et al. prefer inferior pedicle in tumors located superiorly reported $17.6 \%$ of immediate complications (8.1\% skin necrosis, $2.7 \%$ infection, $2.7 \%$ partial NAC necrosis, $1.35 \%$ dehiscence and $1.35 \%$ total NAC necrosis). Obese patients and smokers had a significantly higher rate of complications [14].

The inability to obtain clear margins is a contraindication to breast conservation therapy [15]. The conversion rate to mastectomy in the current study was about 3\% and the conversion was decided after 2 times of involved margin at frozen section. McCully and McMillan reported a series of 50 patients treated with therapeutic mammoplasty in which 4 patients (8\%) required re-operation due to surgical margin involvement [16].

In our study $64.5 \%$ showed excellent esthetic result.
Chang et al. evaluated the degree of satisfaction and cosmetic results, 70\% reported excellent results [11]. Goffman reported on fifty-five patients evaluated by a mixed panel comprised by a surgical oncologist, an oncology nurse, a radiation oncologist and a patient, with $72 \%$ of the evaluations giving excellent and very good results [17].

In the current series the recurrence rate was 3\% and it was distant and not local. Kronowitz reported 5\% of local recurrence after a mean follow-up of 36 months [18]. A slightly lower rate $2 \%$ has been communicated by Losken after a mean follow-up of 40 months, and he recommends that younger patients with extensive ductal carcinoma in situ (DCIS) are poor candidates for simultaneous reconstruction and should, therefore, be deferred until confirmation of negative margins. In his experience, all four patients on whom the oncoplastic approach failed, and who required completion mastectomy with reconstruction, were younger with an extensive DCSI component and, in spite of a negative specimen radiograph test, positive margins were found in a definite pathological study [19].

Goffman reported an $87 \%$ overall rate of local control, $96 \%$ in patients who made it to the point of radiotherapy without local or distant failure, after a median follow-up of 19 months [17].

\section{Conclusion}

Inferior pedicle therapeutic reduction mammoplasty for upper quadrant early breast cancer in large breasted women is a surgically and oncologically safe procedure that yields satisfactory esthetic results with lower morbidity and a desirable improvement in quality of life by reducing their breast size.

\section{REFERENCES}

[1] L. Beloqui and R. M. Rainsbury, "Surgery Insight: Oncoplastic Breast-Conserving Reconstruction-Indications, Benefits, Choices and Outcomes," Nature Clinical Practice Oncology, Vol. 4, No. 11, 2007, pp. 657-664. doi:10.1038/ncponc0957

[2] B. Fisher, S. Anderson, J. Bryant, et al., "Twenty-Year Follow-Up of a Randomized Trial Comparing Total Mastectomy, Lumpectomy, and Lumpectomy Plus Irradiation for the Treatment of Invasive Breast Cancer," New England Journal of Medicine, Vol. 347, No. 16, 2002, pp. 1233-1241. doi:10.1056/NEJMoa022152

[3] A. K. Bajaj, P. S. Kon, K. C. Oberg, et al., "Aesthetic Outcomes in Patients Undergoing Breast Conservation Therapy for Treatment of Localized Breast Cancer,” Plastic and Reconstructive Surgery, Vol. 114, No. 6, 2004, pp. 1442-1449.

[4] M. E. Taylor, C. A. Perez, K. J. Halverson, et al., "Factors Influencing Cosmetic Results after Conservation The- 
rapy for Breast Cancer,” International Journal of Radiation Oncology, Biology, Physics, Vol. 31, No. 4, 1995, pp. 753-764. doi:10.1016/0360-3016(94)00480-3

[5] C. W. Cheng, I. J. Das and B. Stea, "The Effect of the Number of Computed Tommographic Slices on Dose Distributions and Evaluation of Treatment Planning Systems for Radiation Therapy of Intact Breast,” International Journal of Radiation Oncology, Biology, Physics, Vol. 30, No. 1, 1994, pp. 183-195. doi:10.1016/0360-3016(94)90534-7

[6] K. B. Clough, G. J. Kaufman, C. Nos, et al., "Improving Breast Cancer Surgery: A Classification and Quadrant per Quadrant Atlas for Oncoplastic Surgery,” Annals of Surgical Oncology, Vol. 17, No. 5, 2010, pp. 1375-1391. doi:10.1245/s10434-009-0792-y

[7] M. V. Schaverien, C. Raine, E. Majdak-Paredes, et al., “Therapeutic Mammaplasty Extending Indications and Achieving Low Incomplete Excision Rates,” European Journal of Surgical Oncology, 2013, pp. 1-5.

[8] P. L. Giacalone, O. Dubon, P. Roger, et al., "Doughnut Mastopexy Lumpectomy versus Standard Lumpectomy in Breast Cancer Surgery: A Prospective Study,” EJSO, Vol. 33, No. 3, 2007, pp. 301-306.

[9] M. Bouvet, S. E. Singletary, F. Ames, et al., "Combined Breast Conserving Surgery and Bilateral Reduction Mammoplasty Facilitates Postoperative Radiotherapy and Improves Cosmesis in Patients with Early Stage Breast Cancer and Large Pendulous Breast," Breast Disease, Vol. 7, 1996, pp. 236-238.

[10] J. McIntosh and J. M. O’Donoghue, “Therapeutic Mammaplastyea Systematic Review of the Evidence,” European Journal of Surgical Oncology, Vol. 38, No. 3, 2012, pp. 196-202. doi:10.1016/j.ejso.2011.12.004

[11] E. Chang, N. Johnson, B. Webber, et al., "Bilateral Reduction Mammoplasty in Combination with Lumpectomy for Treatment of Breast Cancer in Patients with Macromastia," The American Journal of Surgery, Vol. 187, No. 5, 2004, pp. 647-651. doi:10.1016/j.amjsurg.2004.01.002

[12] M. A. Gulcelik, L. Dogan, M. Camlibel, et al., "Early Com- plications of a Reduction Mammoplasty Technique in the Treatment of Macromastia with or without Breast Cancer,” Clinical Breast Cancer, Vol. 11, No. 6, 2011, pp. 395-399. doi:10.1016/j.clbc.2011.08.001

[13] F. Fitzel, G. Nehrer, M. Deutinger, et al., "Novel Stratigies in Oncoplastic Surgery for Breast Cancer: Immediate Reconstruction of Breast Defects,” European Surgery, Vol. 39, No. 6, 2007, pp. 330-339. doi:10.1007/s10353-007-0373-X

[14] A. M. Munhoz, E. Montag, E. G. Arruda, et al., "Critical Analysis of Reduction Mammaplasty Techniques in Combination with Conservative Breast Surgery for Early Breast Cancer Treatment," Plastic and Reconstructive Surgery, Vol. 117, No. 4, pp. 1091-2003. doi:10.1097/01.prs.0000202121.84583.0d

[15] G. F. Schwartz, U. Veronesi, K. B. Clough, et al., "The Consensus Conference Committee. Consensus on Breast Conservation," Journal of the American College of Surgeons, Vol. 203, No. 2, 2006, pp. 198-207. doi:10.1016/j.jamcollsurg.2006.04.009

[16] S. Mcculley and R. Macmillan, "Therapeutic Mammoplasty Analysis of 50 Consecutive Cases,” British Journal of Plastic Surgery, Vol. 58, No. 7, 2005, pp. 902-907. doi:10.1016/j.bjps.2005.03.007

[17] T. E. Goffman, H. Scheneider, K. Hay, et al., "Cosmesis with Bilateral Mammoreduction for Conservative Breast Cancer Treatment,” Breast Journal, Vol. 11, No. 3, 2005, pp. 195-198. doi:10.1111/j.1075-122X.2005.21610.x

[18] S. J. Kronowitz, K. K. Hunt, H. M. Kuerer, et al., "Practical Guidelines for Repair of Partial Mastectomy Defects Using the Breast Reduction Technique in Patients Undergoing Breast Conservation Therapy," Plastic and Reconstructive Surgery, Vol. 120, No. 7, 2007, pp. 1755-1768. doi:10.1097/01.prs.0000287130.77835.f6

[19] A. Losken, T. M. Styblo, G. W. Carlson, et al., "Management Algorithm and Outcome Evaluation of Partial Mastectomy Defects Treated Using Reduction or Mastopexy Techniques," Annals of Plastic Surgery, Vol. 59, No. 3, 2007, pp. 235-242. doi:10.1097/SAP.0b013e31802ec6d1 